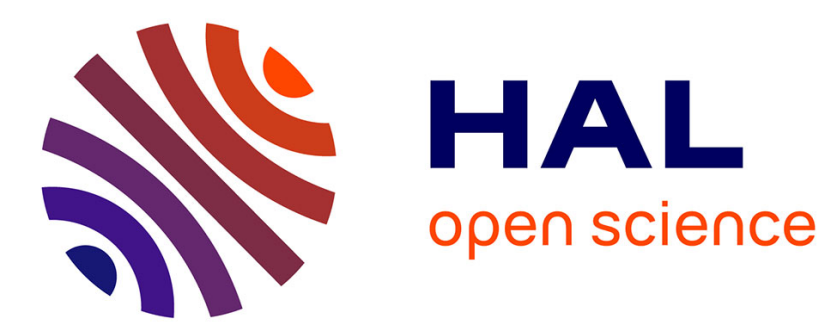

\title{
On the Performance of Space Shift Keying MIMO Systems Over Correlated Rician Fading Channels
}

\author{
Marco Di Renzo, Harald Haas
}

\section{To cite this version:}

Marco Di Renzo, Harald Haas. On the Performance of Space Shift Keying MIMO Systems Over Correlated Rician Fading Channels. IEEE International ITG Workshop on Smart Antennas, Feb 2010, Germany. pp. 1-8. hal-00547044

\section{HAL Id: hal-00547044 \\ https://hal.science/hal-00547044}

Submitted on 15 Dec 2010

HAL is a multi-disciplinary open access archive for the deposit and dissemination of scientific research documents, whether they are published or not. The documents may come from teaching and research institutions in France or abroad, or from public or private research centers.
L'archive ouverte pluridisciplinaire HAL, est destinée au dépôt et à la diffusion de documents scientifiques de niveau recherche, publiés ou non, émanant des établissements d'enseignement et de recherche français ou étrangers, des laboratoires publics ou privés. 


\section{On the Performance of Space Shift Keying MIMO Systems Over Correlated Rician Fading Channels}

\author{
Marco Di Renzo \\ French National Center for Scientific Research (CNRS) \\ Laboratory of Signals and Systems (LSS) \\ École Supérieure d'Électricité (SUPÉLEC) \\ 3 rue Joliot-Curie, 91192 Gif-sur-Yvette (Paris), France \\ E-Mail: marco.direnzo@1ss.supelec.fr
}

\author{
Harald Haas \\ The University of Edinburgh \\ Institute for Digital Communications (IDCOM) \\ College of Science and Engineering \\ Mayfield Road, Edinburgh, EH9 3JL, UK \\ E-Mail: h.haas@ed.ac.uk
}

\begin{abstract}
In this paper, we study the performance of Space Shift Keying (SSK) modulation for a generic Multiple-InputMultiple-Output (MIMO) wireless system over correlated Rician fading channels. In particular, we propose a very general framework for computing the Average Bit Error Probability (ABEP) of SSK-MIMO systems over a generic Rician fading channel with arbitrary correlation and channel parameters. The framework relies upon the Moschopoulos method. We will show that it is exact for MIMO systems with two transmit-antennas and arbitrary receive-antennas, while an asymptotically-tight upper-bound is proposed to handle the system setup with an arbitrary number of transmit-antennas. Analytical frameworks and theoretical findings will be substantiated via Monte Carlo simulations for various system setups.
\end{abstract}

\section{INTRODUCTION}

Spatial Modulation (SM) has been recently proposed as a new and promising candidate transmission technique for low-complexity implementations of Multiple-Input-MultipleOutput (MIMO) wireless systems, which can guarantee a data rate that increases logarithmically with the number of transmit-antennas regardless of the number of receiveantennas [1], [2]. In particular, it has been shown that SM is an excellent solution to solve four main issues amongst the adoption of MIMO systems for low-complexity and powerefficient applications, i.e., i) inter-channel interference, ii) inter-antenna synchronization, iii) multiple radio frequency chains at the transmitter, and iv) the need to have a number of receive-antennas greater than (or equal to) the number of transmit-antennas for some implementations [3]-[5]. Numerical results in [3], [5] have shown that SM can offer better performance and a reduced computational complexity than other popular MIMO schemes over fading channels. Furthermore, in [5] a low-complexity implementation of SM, which is called Space Shift Keying (SSK) modulation, has been suggested and studied. SSK modulation offers a good solution to trade-off receiver complexity for data rate.

The working principle of SM for MIMO systems is based on three main fundamental assumptions: i) the wireless environment naturally modulates the transmitted signal, ii) each transmit-receive wireless link has a different channel, and iii) the receiver employs the a priori channel knowledge to detect the transmitted signal. In other words, SM takes advantage of the location-specific property of the wireless channel, i.e., the uniqueness of each transmit-receive wireless link, for communication [1], [6]. This property is exploited by adopting a simple but effective coding mechanism that establishes a one-to-one mapping between blocks of information bits to be transmitted and the spatial positions of the transmit-antennas in the antenna-array [3]. More specifically, the basic idea of SM is to map a block of information bits into two information carrying units: 1) a symbol that is chosen from a complex signal-constellation diagram, and 2) a unique transmit-antenna index that is chosen from the set of transmit-antennas in the antenna-array. SM allows just one transmit-antenna to be switched on for data transmission and keeps all other transmit-antennas silent at any time instant. Thus, SM entirely avoids inter-channel interference, requires no synchronization among the transmit-antennas, and needs only one radio frequency chain for data transmission: these features make it well-suited for low-complexity MIMO implementations. Moreover, conveying part of the information in the transmit-antenna index introduces a spatial multiplexing gain with respect to single-antenna systems [3].

Owing to its peculiar working principle, SM is a very new transmission technique for data communication over wireless channels. However, research in this field is still at its infancy and fundamental issues still need to be addressed to assess the possibilities of exploitation of this technology over realistic propagation environments and for practical operating conditions. In particular, in the current technical literature there is a lack of sound communication-theoretic frameworks for the analysis, design, and optimization of this novel transmission technology. Some notable results available to date are as follows: i) in [3], the authors have proposed an approximated semi-analytical framework to compute the Average Bit Error Probability (AEBP) of an ad hoc receiver design over independent and identically distributed (i.i.d.) Rayleigh fading channels; ii) the latter framework has been extended to correlated Nakagami- $m$ fading channels in [7]. However, fading correlation is taken into account only for data detection, while the probability of transmit-antenna detection is computed by using the framework in [3], which neglects fading correlation; iii) in [4], [5] the ABEP of the Maximum-Likelihood (ML) 
detector for SM and SSK modulation has been computed for i.i.d. Rayleigh fading channels, respectively; iv) the framework in [5] has been generalized, for the same channel model, in [8] by assuming that more than one antenna can be switched on for data transmission; and v) in [9], [10], we have recently computed the ABEP of SSK modulation over correlated and non-identically distributed Nakagami- $m$ fading channels for optimal and sub-optimal detectors, respectively. However, these latter frameworks are limited to MIMO systems with just one receive-antenna.

In the depicted context, the novel contribution of this paper can be summarized as follows: We propose a general analytical framework to compute the ABEP of SSK-MIMO systems over Rician fading channels with arbitrary channel correlation and fading parameters. The ML-optimum detector in [5] is considered. It is shown that the framework is useful for MIMO systems with arbitrary transmit- and receive-antennas. In particular, by exploiting the Moschopoulos method [11], it is shown that an exact closed-form expression of the ABEP can be derived when the transmitter is equipped with two antennas, while a tight upper-bound can be obtained for arbitrary transmit-antennas. This framework overcomes all restrictions of previously proposed frameworks on either the channel model [3]-[7] or the number of antennas at the receiver [9], [10]. In particular, channel correlation and power imbalance are taken into account.

The remainder of this paper is organized as follows. In Section II, system and channel models are introduced. In Section III, the analytical framework for performance analysis of SSK-MIMO systems over correlated Rician fading channels is developed. In Section IV, numerical and simulation results are shown to substantiate the accuracy of the analytical framework, and to study the performance of SSK modulation over generalized fading channels. Finally, Section V concludes the paper.

\section{SYSTEM MODEL}

Let us consider a generic $N_{t} \times N_{r}$ MIMO system, with $N_{t}$ and $N_{r}$ being the number of transmit- and receive-antennas, respectively. SSK modulation works as follows [3], [5]: i) the transmitter encodes blocks of $\log _{2}\left(N_{t}\right)$ data bits into the index of a single transmit-antenna (i.e., antenna-index coded modulation), which is switched on for data transmission while all other antennas are kept silent, and ii) the receiver solves a $N_{t}$-hypothesis detection problem to estimate the transmitantenna that is not idle, which results in the estimation of the unique sequence of bits emitted by the encoder. Throughout this paper, the unique block of bits encoded into the index of the $i$-th transmit-antenna is called "message" and is denoted by $m_{i}$ for $i=1,2, \ldots, N_{t}$. The $N_{t}$ messages are assumed to be emitted with equal probability by the encoder. Moreover, the related transmitted signal is denoted by $s_{i}(\cdot)$ for $i=$ $1,2, \ldots, N_{t}$. It is implicitly assumed in this notation that, if $m_{i}$ is transmitted, the analog signal $s_{i}(\cdot)$ is emitted by the $i-$ th transmit-antenna while all other transmit-antennas radiate no power.

\section{A. Notation}

Let us briefly introduce the main notation used in what follows. i) We adopt a complex-envelope signal representation. ii) $j=\sqrt{-1}$ is the imaginary unit. iii) $(\cdot)^{*}$ and $(\cdot)^{T}$ denote complex-conjugate and transpose operators, respectively. iv) $(x \otimes y)(t)=\int_{-\infty}^{+\infty} x(\xi) y(t-\xi) d \xi$ is the convolution of signals $x(\cdot)$ and $y(\cdot)$. v) $|\cdot|^{2}$ denotes square absolute value. vi) $\mathrm{E}\{\cdot\}$ is the expectation operator. vii) $\operatorname{Re}\{\cdot\}$ and $\operatorname{Im}\{\cdot\}$ denote the real and imaginary part operators, respectively. viii) $\operatorname{Pr}\{\cdot\}$ means probability. ix) $Q(x)=$ $(1 / \sqrt{2 \pi}) \int_{x}^{+\infty} \exp \left(-t^{2} / 2\right) d t$ is the Q-function. $\left.\mathrm{x}\right) \hat{m}$ denotes the message estimated at the receiver-side. xi) $E_{m}$ is the average energy transmitted by each antenna that emits a non-zero signal. xii) $T_{m}$ denotes the signaling interval for each information message $m_{i}$ for $i=1,2, \ldots, N_{t}$. xiii) The noise $n_{l}$ at the input of the $l$-th receive-antenna $\left(l=1,2, \ldots, N_{r}\right)$ is assumed to be an Additive White Gaussian Noise (AWGN) process, with both real and imaginary parts having a doublesided power spectral density equal to $N_{0}$. Across the receiveantennas, the noises $n_{l}$ for $l=1,2, \ldots, N_{r}$ are statistically independent. xiv) For ease of notation, we set $\bar{\gamma}=E_{m} /\left(4 N_{0}\right)$. xv) $X \sim \mathcal{N}\left(\mu, \sigma^{2}\right)$ denotes a Gaussian Random Variable (RV) with mean $\mu$ and standard deviation $\sigma$. xvi) Matrices and vectors are denoted in boldface. xvii) $\Gamma(\cdot)$ is the Gamma function [12, Eq. (6.1.1)]. xviii) $\delta(\cdot)$ is the Dirac delta function. xix) $G_{p, q}^{m, n}\left(. \mid \begin{array}{c}\left(a_{p}\right) \\ \left(b_{q}\right)\end{array}\right)$ is the Meijer-G function defined in [13, Ch. 8, pp. 519]. xx) $f_{X}(\cdot)$ and $M_{X}(s)=\mathrm{E}\{\exp (-s X)\}$ denote Probability Density Function (PDF) and Moment Generating Function (MGF) of RV X, respectively.

\section{B. Channel Model}

We consider a frequency-flat slowly-varying channel with generically correlated and non-identically distributed Rician fading over the transmit-receive wireless links. In particular:

- $h_{i, l}(t)=\alpha_{i, l} \delta\left(t-\tau_{i, l}\right)$ is the channel impulse response of the transmit-receive wireless link from the $i$-th transmit-antenna to the $l$-th receive-antenna for $i=1,2, \ldots, N_{t}$ and $l=1,2, \ldots, N_{r} . \alpha_{i, l}$ and $\tau_{i, l}$ are the complex channel gains and time-delays, respectively.

- The delays $\tau_{i, l}$ for $i=1,2, \ldots, N_{t}$ and $l=1,2, \ldots, N_{r}$ are assumed to be i.i.d. in $\left[0, T_{m}\right)$, but known at the receiver, i.e., perfect time-synchronization is considered.

- The complex channel gains $\alpha_{i, l}$ for $i=1,2, \ldots, N_{t}$ and $l=1,2, \ldots, N_{r}$ are defined as $\alpha_{i, l}=\beta_{i, l} \exp \left(j \varphi_{i, l}\right)=$ $\alpha_{i, l}^{R}+j \alpha_{i, l}^{I}$, where $\alpha_{i, l}^{R}=\operatorname{Re}\left\{\alpha_{i, l}\right\}, \alpha_{i, l}^{I}=\operatorname{Im}\left\{\alpha_{i, l}\right\}$, $\beta_{i, l}=\sqrt{\left(\alpha_{i, l}^{R}\right)^{2}+\left(\alpha_{i, l}^{I}\right)^{2}}$ is the fading envelope, and $\varphi_{i, l}=\arctan \left(\alpha_{i, l}^{I} / \alpha_{i, l}^{R}\right)$ is the channel phase.

- By assuming a Rician fading channel model [14], we have $\alpha_{i, l}^{R} \sim \mathcal{N}\left(\mu_{i, l}, \sigma_{i, l}^{2}\right)$ and $\alpha_{i, l}^{I} \sim \mathcal{N}\left(\mu_{i, l}, \sigma_{i, l}^{2}\right)$ for $i=1,2, \ldots, N_{t}$ and $l=1,2, \ldots, N_{r}$. Accordingly, $K_{R}^{(i, l)}=\mu_{i, l}^{2} /\left(2 \sigma_{i, l}^{2}\right)$ and $\Omega_{i, l}=\mu_{i, l}^{2}+2 \sigma_{i, l}^{2}$ are the Rice 
factor and the normalized ${ }^{1}$ average Signal-to-NoiseRatio (SNR) of the transmit-receive wireless link from the $i$-th transmit-antenna to the $l$-th receive-antenna for $i=1,2, \ldots, N_{t}$ and $l=1,2, \ldots, N_{r}$, respectively. In particular, $K_{R}^{(i, l)}$ and $\Omega_{i, l}$ for $i=1,2, \ldots, N_{t}$ and $l=1,2, \ldots, N_{r}$ might not be the same over different transmit-receive wireless links.

- A generic channel correlation model is assumed. In particular, the correlation coefficient between the transmitreceive wireless link from the $i_{1}$-th transmit-antenna to the $l_{1}$-th receive-antenna for $i_{1}=1,2, \ldots, N_{t}$ and $l_{1}=1,2, \ldots, N_{r}$, and the transmit-receive wireless link from the $i_{2}$-th transmit-antenna to the $l_{2}$-th receiveantenna for $i_{2}=1,2, \ldots, N_{t}$ and $l_{2}=1,2, \ldots, N_{r}$ is as follows:

$\rho_{\left(i_{1}, i_{2}\right)}^{A B}\left(l_{1}, l_{2}\right)=\frac{\mathrm{E}\left\{\left(\alpha_{i_{1}, l_{1}}^{A}-\mu_{i_{1}, l_{1}}\right)\left(\alpha_{i_{2}, l_{2}}^{B}-\mu_{i_{2}, l_{2}}\right)\right\}}{\sigma_{i_{1}, l_{1}} \sigma_{i_{2}, l_{2}}}$

where $A=\{R, I\}$ and $B=\{R, I\}$ account for the real and imaginary components of the complex channel gains. Given a pair of transmit-antennas $\left(i_{1}, i_{2}\right)$ for $i_{1}=$ $1,2, \ldots, N_{t}$ and $i_{2}=1,2, \ldots, N_{t}$, we denote by $\boldsymbol{\Sigma}_{\left(i_{1}, i_{2}\right)}^{A B}$ the covariance matrix whose element in the $l_{1}$-th row and $l_{2}-$ th column is as follows:

$$
\Sigma_{\left(i_{1}, i_{2}\right)}^{A B}\left(l_{1}, l_{2}\right)=\mathrm{E}\left\{\left(\alpha_{i_{1}, l_{1}}^{A}-\mu_{i_{1}, l_{1}}\right)\left(\alpha_{i_{2}, l_{2}}^{B}-\mu_{i_{2}, l_{2}}\right)\right\}
$$

Moreover, we have:

$$
\begin{array}{r}
\sigma_{i, l}^{2}=\mathrm{E}\left\{\left(\alpha_{i, l}^{R}-\mu_{i, l}\right)\left(\alpha_{i, l}^{R}-\mu_{i, l}\right)\right\} \\
=\mathrm{E}\left\{\left(\alpha_{i, l}^{I}-\mu_{i, l}\right)\left(\alpha_{i, l}^{I}-\mu_{i, l}\right)\right\} \\
\mathrm{E}\left\{\left(\alpha_{i, l}^{R}-\mu_{i, l}\right)\left(\alpha_{i, l}^{I}-\mu_{i, l}\right)\right\}=0
\end{array}
$$

for $i=1,2, \ldots, N_{t}$ and $l=1,2, \ldots, N_{r}$ due to the assumption of Rician fading.

\section{ABEP OVER CORRELATED RICIAN FADING CHANNELS}

Let $m_{n}$ with $n=1,2, \ldots, N_{t}$ be the transmitted message. The signal received after propagation through the wireless fading channel and impinging upon the $l$-th receive-antenna can be written as follows $\left(l=1,2, \ldots, N_{r}\right)$ :

$$
r_{l}(t)=\tilde{s}_{n, l}(t)+n_{l}(t) \quad \text { if } m_{n} \text { is sent }
$$

where $\tilde{s}_{n, l}(t)=\left(s_{n} \otimes h_{n, l}\right)(t)=\alpha_{n, l} s_{n}\left(t-\tau_{n, l}\right)=$ $\beta_{n, l} \exp \left(j \varphi_{n, l}\right) s_{n}\left(t-\tau_{n, l}\right)$ with $n=1,2, \ldots, N_{t}$ and $l=$ $1,2, \ldots, N_{r}$.

In particular, (5) is a general $N_{t}$-hypothesis detection problem [14, Sec. 7.1], [15, Sec. 4.2, pp. 257] in AWGN, when conditioning upon fading channel statistics. Accordingly, the ML-optimum detector with full Channel State Information (CSI) and perfect time-synchronization at the receiver is as

\footnotetext{
${ }^{1} \Omega_{i, l}$ is normalized to the transmit-energy $\left(E_{m}\right)$ and the noise power $\left(N_{0}\right)$ at the receiver input.
}

follows [4], [14, Sec. 7.1]:

$$
\hat{m}=\underset{m_{i} \text { for } i=1,2, \ldots, N_{t}}{\arg \max }\left\{D_{i}\right\}
$$

where $D_{i}$ for $i=1,2, \ldots, N_{t}$ are the decision metrics defined in what follows:

$$
\begin{aligned}
D_{i} & =\sum_{l=1}^{N_{r}}\left[\operatorname{Re}\left\{\int_{T_{m}} r_{l}(t) \tilde{s}_{i, l}^{*}(t) d t\right\}\right] \\
& -\frac{1}{2} \sum_{l=1}^{N_{r}}\left[\int_{T_{m}} \tilde{s}_{i, l}(t) \tilde{s}_{i, l}^{*}(t) d t\right]
\end{aligned}
$$

If the transmitted message is $m_{n}$, which results in switching on the $n$-th transmit-antenna for data transmission, the detector will be successful in detecting the transmitted message, i.e., $\hat{m}=m_{n}$, if and only if $\max _{i=1,2, \ldots, N_{t}}\left\{D_{i}\right\}=D_{n}$.

SSK modulation in [3]-[5] implicitly assumes that the signal transmitted by the $n$-th transmit-antenna is $s_{n}(t)=$ $\sqrt{E_{m}} w(t) \forall n=1,2, \ldots, N_{t}$, where $w(\cdot)$ denotes the unitenergy (i.e., $\int_{-\infty}^{+\infty}|w(t)|^{2} d t=1$ ) elementary pulse waveform for each transmission. Accordingly, the signal impinging upon the $l$-th receive-antenna is as follows $\left(l=1,2, \ldots, N_{r}\right)$ :

$$
r_{l}(t)=\sqrt{E_{m}} \beta_{n, l} \exp \left(j \varphi_{n, l}\right) w(t)+n_{l}(t)
$$

We note that in (8) the channel delays $\tau_{n, l}$ for $n=$ $1,2, \ldots, N_{t}$ and $l=1,2, \ldots, N_{r}$ do not appear explicitly. The reason is that (9) implicitly assumes that: either i) a pure sinusoidal tone is transmitted by each antenna, i.e., $w(t)=1$, and the propagation delays are embedded, with a slight abuse of notation, into the channel phases, or ii) $\tau_{1,1} \cong \tau_{1,2} \cong \ldots \cong$ $\tau_{N_{t}, N_{r}}$, which is a realistic assumption when the distance between the transmitter and the receiver is much larger than the spacing between the transmit- and receive-antennas, and, to a first-order, the signals transmitted by the antennas differ only in phase [16, Eq. (7.24)], which can be still embedded into the channel phases. In this latter case, the delays can be neglected in the light of the assumption of perfect time-synchronization at the receiver.

From (8), the decision metrics in (7), when conditioned upon the transmission of message $m_{n}$, i.e., $D_{i \mid m_{n}}$, can be written as follows $\left(n=1,2, \ldots, N_{t}\right.$ and $\left.i=1,2, \ldots, N_{t}\right)$ :

$D_{i \mid m_{n}}=\sum_{l=1}^{N_{r}}\left[\operatorname{Re}\left\{\alpha_{n, l} \alpha_{i, l}^{*}\right\} E_{m}+\beta_{i, l} \sqrt{E_{m}} \tilde{n}_{i, l}-\frac{1}{2} \beta_{i, l}^{2} E_{m}\right]$

where $\tilde{n}_{i, l}=\operatorname{Re}\left\{\int_{T_{m}} n_{l}(t) \exp \left(-j \varphi_{i, l}\right) w^{*}(t) d t\right\}$.

\section{A. The $2 \times N_{r}$ MIMO Case}

1) Conditional Error Probability: Let us consider $N_{t}=$ 2. From the decision rule in (6), the probability of wrong detecting the index of the transmit-antenna, $\mathrm{P}_{\mathrm{E}}(\cdot, \cdot)$, when conditioning upon the channel impulse responses $\mathbf{h}_{1}=$ $\left[h_{1,1}, h_{1,2}, \ldots, h_{1, N_{r}}\right]$ and $\mathbf{h}_{2}=\left[h_{2,1}, h_{2,2}, \ldots, h_{2, N_{r}}\right]$, can 


$$
\left\{\begin{array}{l}
\Psi_{2,1}=\prod_{h=1}^{L}\left[\frac{\chi_{1}^{(2,1)}}{\chi_{h}^{(2,1)}} \exp \left(-\frac{\kappa_{h}^{(2,1)}}{\chi_{h}^{(2,1)}}\right)\right] \\
\vartheta_{k}^{(2,1)}=\frac{1}{k} \sum_{r=1}^{k}\left\{\sum_{b=1}^{L}\left[v_{r}^{(2,1)}\left(1-\frac{\chi_{1}^{(2,1)}}{\chi_{b}^{(2,1)}}\right)^{r}+\frac{r \chi_{1}^{(2,1)} \kappa_{r}^{(2,1)}}{\left(\chi_{b}^{(2,1)}\right)^{2}}\left(1-\frac{\chi_{1}^{(2,1)}}{\chi_{b}^{(2,1)}}\right)^{r-1}\right] \vartheta_{k-r}^{(2,1)}\right\} \quad k \geq 1
\end{array}\right.
$$

be explicitly written as follows:

$$
\begin{aligned}
\mathrm{P}_{\mathrm{E}}\left(\mathbf{h}_{1}, \mathbf{h}_{2}\right) & =\frac{1}{2} \operatorname{Pr}\left\{D_{1 \mid m_{1}}<D_{2 \mid m_{1}}\right\} \\
& +\frac{1}{2} \operatorname{Pr}\left\{D_{2 \mid m_{2}}<D_{1 \mid m_{2}}\right\}
\end{aligned}
$$

After a few algebraic manipulations, $\mathrm{P}_{\mathrm{E}}(\cdot, \cdot)$ in $(10)$ can be written as follows:

$$
\mathrm{P}_{\mathrm{E}}\left(\mathbf{h}_{1}, \mathbf{h}_{2}\right)=\operatorname{Pr}\left\{E_{m} \sum_{l=1}^{N_{r}}\left|\alpha_{2, l}-\alpha_{1, l}\right|^{2}<\tilde{n}\right\}
$$

where we have defined $\tilde{n}=\sum_{l=1}^{N_{r}} \tilde{n}_{l}$ with $\tilde{n}_{l}=$ $2 \beta_{2, l} \sqrt{E_{m}} \tilde{n}_{2, l}-2 \beta_{1, l} \sqrt{E_{m}} \tilde{n}_{1, l}$. In particular, when conditioning upon the fading channel gains, $\tilde{n}$ is a Gaussian distributed RV with zero-mean and variance $\mathrm{E}\left\{\tilde{n}^{2}\right\}=$ $4 N_{0} E_{m} \sum_{l=1}^{N_{r}}\left|\alpha_{2, l}-\alpha_{1, l}\right|^{2}$. Furthermore, we have taken into account that, due to symmetry, $\operatorname{Pr}\left\{D_{1 \mid m_{1}}<D_{2 \mid m_{1}}\right\}=$ $\operatorname{Pr}\left\{D_{2 \mid m_{2}}<D_{1 \mid m_{2}}\right\}$.

From $(11), \mathrm{P}_{\mathrm{E}}(\cdot, \cdot)$ can be readily computed in closed-form as follows [15, Sec. 2.2.2]:

$$
\mathrm{P}_{\mathrm{E}}\left(\mathbf{h}_{1}, \mathbf{h}_{2}\right)=Q\left(\sqrt{\bar{\gamma} \sum_{l=1}^{N_{r}}\left|\alpha_{2, l}-\alpha_{1, l}\right|^{2}}\right)
$$

2) Statistics of the End-to-End SNR: To compute the ABEP, the conditional error probability in (12) needs to be averaged over $\left(\mathbf{h}_{1}, \mathbf{h}_{2}\right)$, i.e., $\mathrm{ABEP}_{2,1}=\mathrm{E}\left\{\mathrm{P}_{\mathrm{E}}\left(\mathbf{h}_{1}, \mathbf{h}_{2}\right)\right\}$. We exploit the Moschopoulos method [11] to get a closed-form expression of the ABEP over generically correlated Rician fading channels. To this end, some preliminary considerations are required. In particular, from Section II-B we have $(i=$ $1,2, \ldots, N_{t}$ and $\left.l=1,2, \ldots, N_{r}\right)$ :

$$
\begin{aligned}
\Xi_{l}^{(2,1)} & =\alpha_{2, l}-\alpha_{1, l}=\left(\alpha_{2, l}^{R}+j \alpha_{2, l}^{I}\right)-\left(\alpha_{1, l}^{R}+j \alpha_{1, l}^{I}\right) \\
& =\left(\alpha_{2, l}^{R}-\alpha_{1, l}^{R}\right)+j\left(\alpha_{2, l}^{I}-\alpha_{1, l}^{I}\right)=\xi_{R, l}^{(2,1)}+j \xi_{I, l}^{(2,1)}
\end{aligned}
$$

with $\xi_{R, l}^{(2,1)}=\alpha_{2, l}^{R}-\alpha_{1, l}^{R}$ and $\xi_{I, l}^{(2,1)}=\alpha_{2, l}^{I}-\alpha_{1, l}^{I}$.

From the channel model in Section II-B, it can be readily shown that the $2 N_{r} \times 1$ column vector $\boldsymbol{\Xi}^{(2,1)}=$ $\left[\xi_{R, 1}^{(2,1)}, \xi_{R, 2}^{(2,1)}, \ldots, \xi_{R, N_{r}}^{(2,1)}, \xi_{I, 1}^{(2,1)}, \xi_{I, 2}^{(2,1)}, \ldots, \xi_{I, N_{r}}^{(2,1)}\right]^{T}$ is Gaussian distributed with mean vector, $\boldsymbol{\mu}_{\boldsymbol{\Xi}^{(2,1)}}$, and covariance matrix, $\boldsymbol{\Sigma}_{\boldsymbol{\Xi}^{(2,1)}}$, defined in what follows, respectively:

$$
\begin{gathered}
\boldsymbol{\mu}_{\boldsymbol{\Xi}^{(2,1)}}=\left[\left(\boldsymbol{\mu}_{2}^{R}-\boldsymbol{\mu}_{1}^{R}\right)^{T},\left(\boldsymbol{\mu}_{2}^{I}-\boldsymbol{\mu}_{1}^{I}\right)^{T}\right]^{T} \\
\boldsymbol{\Sigma}_{\boldsymbol{\Xi}^{(2,1)}}=\left[\begin{array}{cc}
\boldsymbol{\Sigma}_{\Xi^{(2,1)}}^{R R} & \boldsymbol{\Sigma}_{\boldsymbol{\Xi}^{(2,1)}}^{R I} \\
\left(\boldsymbol{\Sigma}_{\boldsymbol{\Xi}^{(2,1)}}^{R I}\right)^{T} & \boldsymbol{\Sigma}_{\Xi^{(2,1)}}^{I I}
\end{array}\right]
\end{gathered}
$$

where $\boldsymbol{\mu}_{i}^{R}=\boldsymbol{\mu}_{i}^{I}=\left[\mu_{i, 1}, \mu_{i, 2}, \ldots, \mu_{i, N_{r}}\right]^{T}$ for $i=1,2$ are $N_{r} \times 1$ vectors, and $\boldsymbol{\Sigma}_{\Xi^{(2,1)}}^{A B}$ for $A=\{R, I\}$ and $B=\{R, I\}$ are $N_{r} \times N_{r}$ matrices whose element in the $p$-th row and $q-$ th column is $\boldsymbol{\Sigma}_{\Xi^{(2,1)}}^{A B}(p, q)=\boldsymbol{\Sigma}_{(2,2)}^{A B}(p, q)-\boldsymbol{\Sigma}_{(2,1)}^{A B}(p, q)-$ $\boldsymbol{\Sigma}_{(1,2)}^{A B}(p, q)+\boldsymbol{\Sigma}_{(1,1)}^{A B}(p, q)$ for $p=1,2, \ldots, N_{r}$ and $q=$ $1,2, \ldots, N_{r}$.

3) Moschopoulos Method: From (13)-(15), it follows that $\gamma_{2,1}=\sum_{l=1}^{N_{r}}\left|\alpha_{2, l}-\alpha_{1, l}\right|^{2}$ in (12) is the summation of the absolute square value of $N_{r}$ complex Gaussian RVs, with known mean vector (14) and covariance matrix (15). As a consequence, the MGF, $M_{\gamma_{2,1}}(\cdot)$, and the PDF, $f_{\gamma_{2,1}}(\cdot)$, of $\gamma_{2,1}$ can be computed in closed-form by exploiting the general result in [17, Theorem 1]. In particular, the MGF is equal to [17, Eq. (25)]:

$M_{\gamma_{2,1}}(s)=\prod_{h=1}^{L}\left[\left(1+\chi_{h}^{(2,1)} s\right)^{-v_{h}^{(2,1)}} \exp \left(-\frac{\kappa_{h}^{(2,1)} s}{1+\chi_{h}^{(2,1)} s}\right)\right]$ where $L=2 N_{r}$ and, for $h=1,2, \ldots, L, \chi_{h}^{(2,1)}=$ $2 \lambda_{h}^{(2,1)}, v_{h}^{(2,1)}=1 / 2, \kappa_{h}^{(2,1)}=\left(\eta_{h}^{(2,1)}\right)^{2}, \mathbf{D}^{(2,1)}=$ $\operatorname{diag}\left(\lambda_{1}^{(2,1)}, \lambda_{2}^{(2,1)}, \ldots, \lambda_{L}^{(2,1)}\right)$ is the diagonal matrix of the eigenvalues $\left(\lambda_{1}^{(2,1)}, \lambda_{2}^{(2,1)}, \ldots, \lambda_{L}^{(2,1)}\right)$ of the covariance matrix in (15), $\mathbf{T}^{(2,1)}$ is a $L \times L$ orthogonal matrix containing the orthogonal eigenvectors of the covariance matrix in (15) such that $\boldsymbol{\Sigma}_{\boldsymbol{\Xi}^{(2,1)}}=\mathbf{T}^{(2,1)} \mathbf{D}^{(2,1)}\left(\mathbf{T}^{(2,1)}\right)^{T}$, and $\eta_{h}^{(2,1)}$ is the $h$-th element of the column vector $\boldsymbol{\eta}^{(2,1)}=\left(\mathbf{T}^{(2,1)}\right)^{T} \boldsymbol{\mu}_{\Xi^{(2,1)}}$.

From (16), a closed-form expression of the PDF, $f_{\gamma_{2,1}}(\cdot)$, of $\gamma_{2,1}$ can be obtained from [17, Theorem 1] by using the Moschopoulos method. The final result is as follows:

$f_{\gamma_{2,1}}(\zeta)=\Psi_{2,1} \sum_{k=0}^{+\infty}\left[\vartheta_{k}^{(2,1)} \frac{\zeta^{v^{(2,1)}+k-1} \exp \left(-\frac{\zeta}{\chi_{1}^{(2,1)}}\right)}{\left(\chi_{1}^{(2,1)}\right)^{v^{(2,1)}+k} \Gamma\left(v^{(2,1)}+k\right)}\right]$

where $\Psi_{2,1}$ and $\vartheta_{k}^{(2,1)}$ are defined in (18) on top of this page, and $v^{(2,1)}=\sum_{h=1}^{L} v_{h}^{(2,1)}, \quad \chi_{1}^{(2,1)}=$ $\min \left\{\chi_{h}^{(2,1)} \mid 1 \leq h \leq L\right\}, \vartheta_{0}^{(2,1)}=1$.

4) $M G F-$ and PDF-based Approach to Compute the ABEP: Finally, by using (16) and (17), the $\mathrm{ABEP}_{2,1}$ can be computed from (12) by using either a MGF- or a PDF-based approach [14], as shown in (19) below:

$$
\operatorname{ABEP}_{2,1}=\frac{1}{\pi} \int_{0}^{\pi / 2} M_{\gamma_{2,1}}\left(\frac{\bar{\gamma}}{2 \sin ^{2}(\theta)}\right) d \theta
$$




$$
\mathrm{ABEP}_{2,1}=\int_{0}^{+\infty} Q(\sqrt{\bar{\gamma} \zeta}) f_{\gamma_{2,1}}(\zeta) d \zeta=\frac{\Psi_{2,1}}{2 \sqrt{\pi}} \sum_{k=0}^{+\infty}\left[\frac{\vartheta_{k}^{(2,1)}}{\Gamma\left(v^{(2,1)}+k\right)} G_{2,2}^{2,1}\left(\frac{\bar{\gamma} \chi_{1}^{(2,1)}}{2} \mid \begin{array}{cc}
1-v^{(2,1)}-k & 1 \\
0 & 1 / 2
\end{array}\right)\right]
$$

and (20) on top of this page, respectively, where the last equality in (20) is obtained from the Mellin-Barnes theorem in [13, Eq. (2.24.1.1)].

Both formulas in (19) and (20) offer the same result. However, the integral in (19) cannot, to the best of our knowledge, be computed in closed-form and numerical methods need to be used. This can be done with consolidated numerical methods, for example Gauss-Legendre Quadrature Rules [18]. On the contrary, (20) offers a truly closed-form expression of the ABEP. An efficient method to compute the series in (17) and (20) can be found in [19].

\section{B. The $N_{t} \times N_{r}$ MIMO Case}

The extension of the framework in Section III-A is quite cumbersome when $N_{t}>2$. To avoid this complexity, we take advantage of a recently proposed bound to compute the performance of SSK modulation when $N_{t}>2$. The bound has been proposed in [9] for a system setup with a single receiveantenna and validated for Nakagami- $m$ fading. In [9], it has been shown that the bound can overcome the limitations of other bounds already available in the literature for Rayleigh fading, e.g., [5]. In this paper, we simply use this bound for a generic MIMO system. The interested reader can find all details of the derivation in [9].

By using [9, Eq. (33)], the ABEP of a generic $N_{t} \times N_{r}$ SSKMIMO system over correlated Rician fading channel can be tightly upper-bounded as follows:

$$
\mathrm{ABEP} \leq \mathrm{ABEP}^{\mathrm{B}}=\frac{1}{N_{t}-1} \sum_{i_{1}=1}^{N_{t}} \sum_{i_{2}=i_{1}+1}^{N_{t}} \mathrm{ABEP}_{i_{2}, i_{1}}
$$

where $\mathrm{ABEP}_{i_{2}, i_{1}}$ for $i_{1}=1,2, \ldots, N_{t}$ and $i_{2}=1,2, \ldots, N_{t}$ is the ABEP of an equivalent $2 \times N_{r}$ SSK-MIMO system, which consists of only two transmit-antennas, i.e., the antennas with indexes $i_{1}$ and $i_{2}$. In practice, $\mathrm{ABEP}_{i_{2}, i_{1}}$ can be obtained from (19) and (20) as if the transmit-antennas $i_{1}$ and $i_{2}$ were the only available in the MIMO system. Apart from replacing all indexes in Section III-A, i.e., $(2,1) \rightarrow\left(i_{2}, i_{1}\right)$, all formulas are still valid and can be readily applied.

In Section IV, we will show that the bound in (21), since based on union bound methods [9], is asymptotically tight for high SNRs and can offer a good estimate of the ABEP for the SNRs of practical interest.

\section{Analysis of the Diversity Order}

The diversity order of a generic $N_{t} \times N_{r}$ SSK-MIMO system can be computed by using the unifying parametrization in [20]. For example, by using [20, Proposition 3], which applies to the MGF of the end-to-end SNR when $|s| \rightarrow+\infty$, we can readily compute the diversity gain, $G_{d}$, as follows $G_{d}=\sum_{h=1}^{L} v_{h}^{(\cdot, \cdot)}=\sum_{h=1}^{2 N_{r}}(1 / 2)=N_{r}$. Note that, this result is valid for the scenario with arbitrary transmit-antennas, since the MGF when $N_{t}>2$ is simply the summation of the MGFs of equivalent SSK-MIMO systems with two transmitantennas: this follows from (19) and (21). As obtained in [5] for i.i.d. Rayleigh fading, the diversity gain depends only on the number of receive-antennas, while the number of transmitantennas affects the coding gain only. The coding gain can be readily computed by using the parametrization in [20] as well. However, it is here omitted due to space constrains. This result clearly shows that SSK-MIMO schemes can offer only receive-diversity gains, and cannot exploit the transmitantennas to increase the diversity order.

\section{Numerical and Simulation Results}

In this section, we describe some numerical results aiming at validating the accuracy of the proposed analytical framework and at studying the performance of SSK-MIMO schemes over generalized fading channels.

a) System Setup: For illustrative purposes, the following setup is used. i) The curves are shown as a function of the Rice factor $K_{R}^{(i, l)}$ and the average SNR of the transmitreceive wireless link from the $i$-th transmit-antenna to the $l$-th receive-antenna. The parameters (i.e., mean and standard deviation) of the related Gaussian RVs can be computed as (see Section II-B) $\mu_{i, l}=\sqrt{\Omega_{i, l}\left[K_{R}^{(i, l)} /\left(K_{R}^{(i, l)}+1\right)\right]}$ and $\sigma_{i, l}=\sqrt{\Omega_{i, l} /\left[2\left(K_{R}^{(i, l)}+1\right)\right]}$. ii) As far as the channel correlation model is concerned, we consider the "Kronecker model" [21], [22] with exponential correlation [23]. However, we emphasize here that the analytical framework proposed in Section III can be used for arbitrary correlation matrices, including correlation matrices obtained from measurements. The adoption of a "Kronecker model" is only due to its simplicity of implementation, and we expect that SSK modulation will show similar performance trends for different correlation models. In particular, we assume ${ }^{2} \Sigma_{i_{1}, i_{2}}^{R R}\left(l_{1} \cdot l_{2}\right)=$ $\Sigma_{i_{1}, i_{2}}^{I I}\left(l_{1} . l_{2}\right)=\sigma_{i_{1}, l_{1}} \sigma_{i_{2}, l_{2}} \rho^{\left|i_{1}-i_{2}\right|} \rho^{\left|l_{1}-l_{2}\right|}$ and $\Sigma_{i_{1}, i_{2}}^{R I}\left(l_{1} . l_{2}\right)=$ $\Sigma_{i_{1}, i_{2}}^{I R}\left(l_{1} . l_{2}\right)=0$. Furthermore, $\rho \in[0,1]$ is a constant factor used to study the performance for various correlation coefficients. iii) The numerical values of all parameters can be found in the caption of each figure.

b) Performance of SSK Modulation over Rician Fading - Power Balance: In Figs. 1-4, we show the ABEP of SSK modulation for various combinations of transmit- and receiveantennas over the same Rician fading channel with identically distributed fading. The following trends can be observed. i) We notice that the analytical model developed in Section III

\footnotetext{
${ }^{2}$ Note that with this notation we implicitly assume, with a slight abuse of notation, $\rho^{0}=1$ for all values of $\rho$.
} 


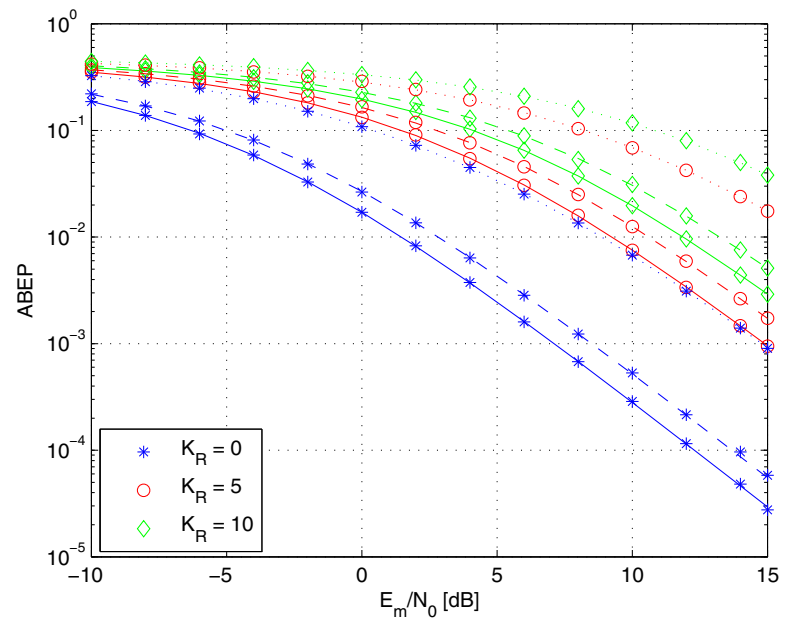

Fig. 1. ABEP against $E_{m} / N_{0}$. Solid, dashed, and dotted lines denote the analytical model in Section III and markers Monte Carlo simulations. Setup: i) $N_{t}=2$, ii) $N_{r}=2$, iii) $\Omega_{i, l}=10 \mathrm{~dB}$ and $K_{R}^{(i, l)}=K_{R}$ for $i=$ $1,2, \ldots, N_{t}$ and $l=1,2, \ldots, N_{r}$, and iv) $\rho=0.00$ (solid lines), $\rho=0.25$ (dashed lines), $\rho=0.75$ (dotted lines).

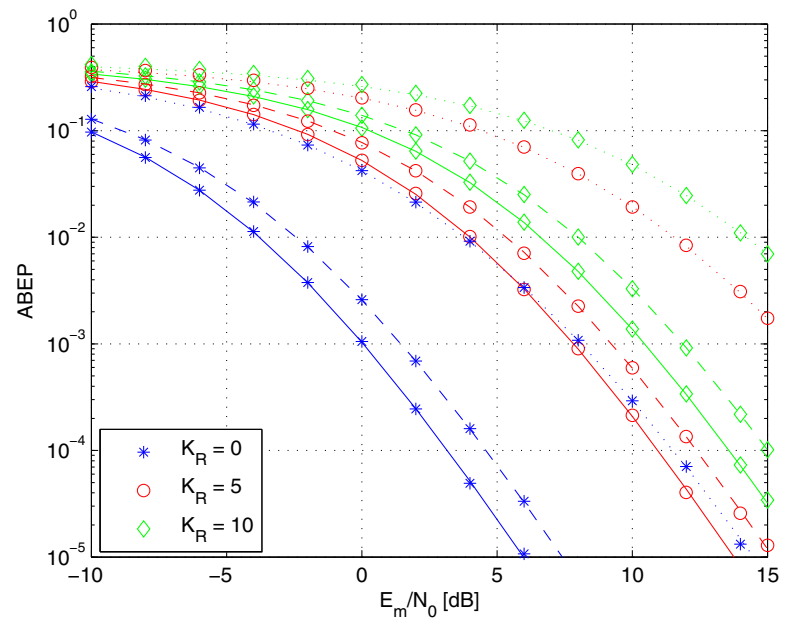

Fig. 2. ABEP against $E_{m} / N_{0}$. Solid, dashed, and dotted lines denote the analytical model in Section III and markers Monte Carlo simulations. Setup: i) $N_{t}=2$, ii) $N_{r}=4$, iii) $\Omega_{i, l}=10 \mathrm{~dB}$ and $K_{R}^{(i, l)}=K_{R}$ for $i=$ $1,2, \ldots, N_{t}$ and $l=1,2, \ldots, N_{r}$, and iv) $\rho=0.00$ (solid lines), $\rho=0.25$ (dashed lines), $\rho=0.75$ (dotted lines).

is very accurate, and the bound for $N_{t}>2$ tightly overlaps with Monte Carlo simulations for ABEP $\leq 10^{-1}$, which is the range of interest for practical applications. ii) The ABEP degrades for increasing values of the Rice factor $K_{R}^{(i, l)}=K_{R}$. The reason is as follows. For increasing values of $K_{R}$ the fading fluctuations tend to be less pronounced and the amount of fading gets smaller [14]. In particular, we have Rayleigh fading if $K_{R}=0$ and no fading if $K_{R} \rightarrow+\infty$. Since the scenario in Figs. 1-4 consider a wireless channel with the same average SNR $\Omega_{i, l}=\Omega$, from (12) and (21) we conclude that the less the amount of fading is, the closer the complex channel gains are, and, so, the worse the ABEP is. This is a very

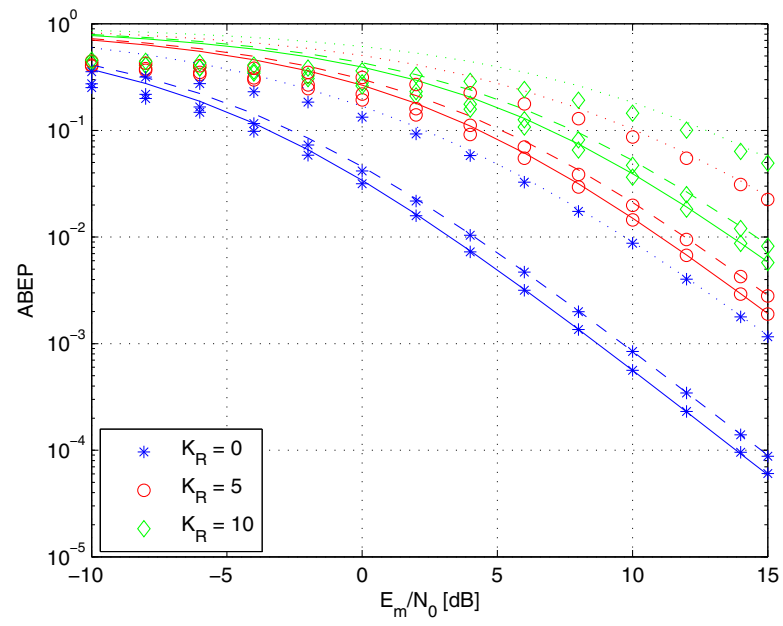

Fig. 3. ABEP against $E_{m} / N_{0}$. Solid, dashed, and dotted lines denote the analytical model in Section III and markers Monte Carlo simulations. Setup: i) $N_{t}=4$, ii) $N_{r}=2$, iii) $\Omega_{i, l}=10 \mathrm{~dB}$ and $K_{R}^{(i, l)}=K_{R}$ for $i=$ $1,2, \ldots, N_{t}$ and $l=1,2, \ldots, N_{r}$, and iv) $\rho=0.00$ (solid lines), $\rho=0.25$ (dashed lines), $\rho=0.75$ (dotted lines).

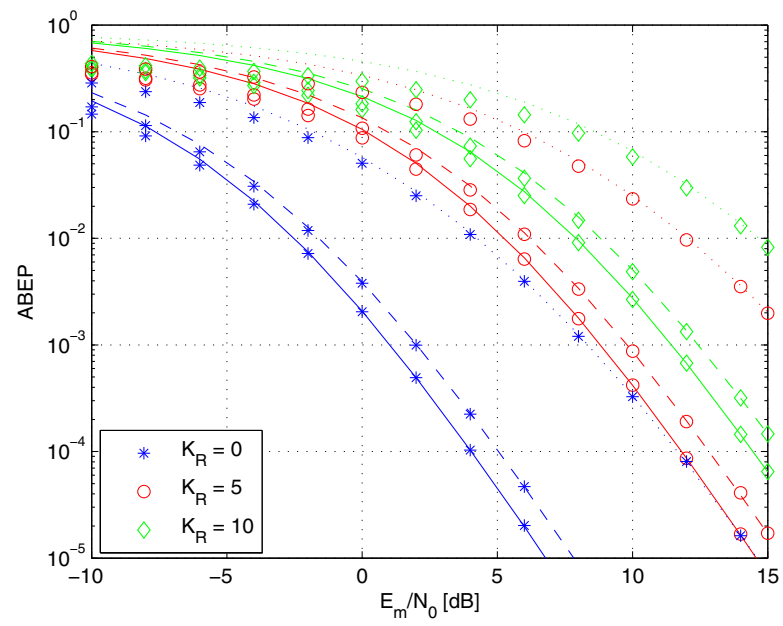

Fig. 4. ABEP against $E_{m} / N_{0}$. Solid, dashed, and dotted lines denote the analytical model in Section III and markers Monte Carlo simulations. Setup: i) $N_{t}=4$, ii) $N_{r}=4$, iii) $\Omega_{i, l}=10 \mathrm{~dB}$ and $K_{R}^{(i, l)}=K_{R}$ for $i=$ $1,2, \ldots, N_{t}$ and $l=1,2, \ldots, N_{r}$, and iv) $\rho=0.00$ (solid lines), $\rho=0.25$ (dashed lines), $\rho=0.75$ (dotted lines).

different behavior with respect to conventional modulation schemes, where, in general, the ABEP improves for less pronounced fading fluctuations [14]. However, this result is inherent in the working mechanism of SSK modulation, which requires the wireless links to be sufficiently different from each other to achieve good performance. iii) By studying the ABEP with respect to the correlation coefficient, we observe that it gets worse for increasing values of the channel correlation, as expected. In particular, we observe that for large values of the correlation coefficient the detector might be unable to distinguish the messages sent by the different transmitantennas. This conclusion also follows from (12) and (21), 


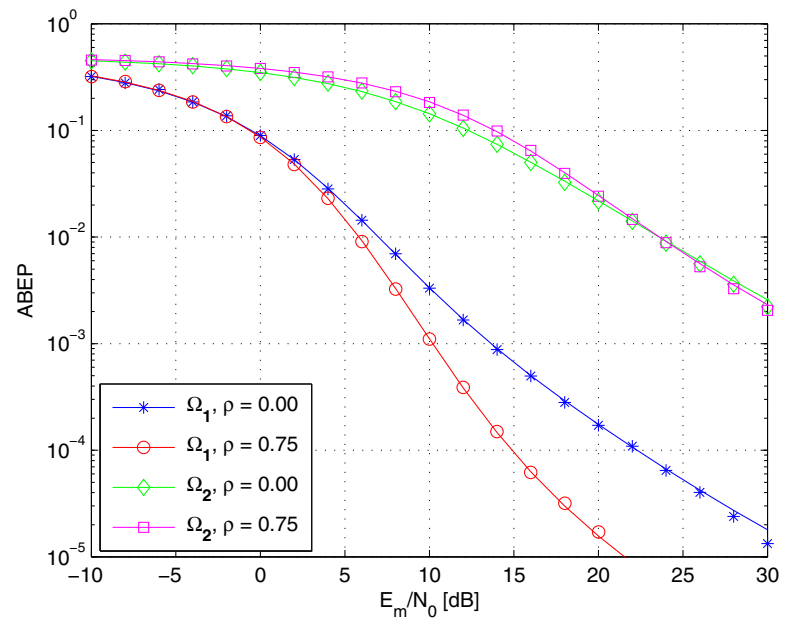

Fig. 5. ABEP against $E_{m} / N_{0}$. Solid lines denote the analytical model in Section III and markers Monte Carlo simulations. Setup: i) $N_{t}=2$, ii) $N_{r}=$ 1, iii) $\boldsymbol{\Omega}_{\mathbf{1}}=\left[\Omega_{1,1}, \Omega_{2,1}\right]=[0,10] \mathrm{dB}, \boldsymbol{\Omega}_{\mathbf{2}}=\left[\Omega_{1,1}, \Omega_{2,1}\right]=[0,3] \mathrm{dB}$, and iv) $K_{R}^{(i, l)}=K_{R}=5 \mathrm{~dB}$ for $i=1,2, \ldots, N_{t}$ and $l=1,2, \ldots, N_{r}$.

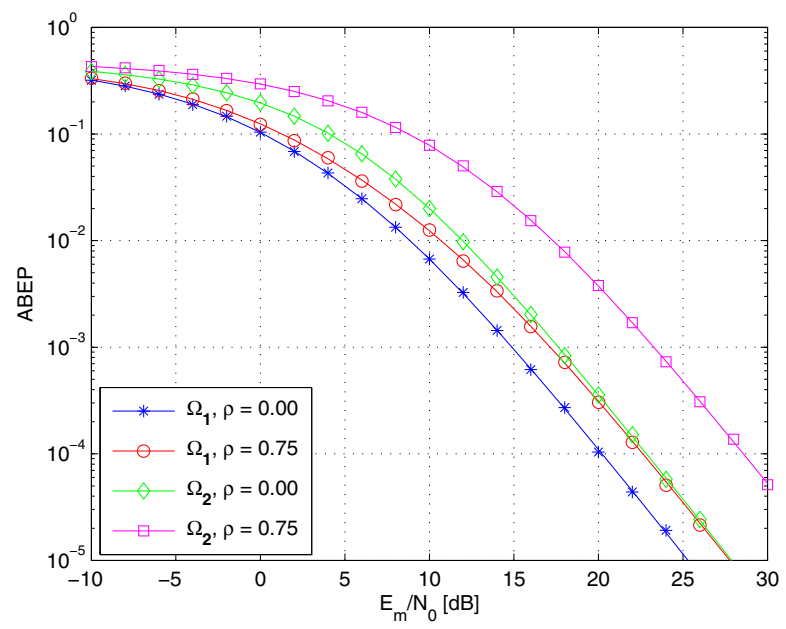

Fig. 6. ABEP against $E_{m} / N_{0}$. Solid lines denote the analytical model in Section III and markers Monte Carlo simulations. Setup: i) $N_{t}=2$, ii) $N_{r}=2$, iii) $\boldsymbol{\Omega}_{\mathbf{1}}=\left[\Omega_{1,1}, \Omega_{1,2}, \Omega_{2,1}, \Omega_{2,2}\right]=[-20,-10,0,10] \mathrm{dB}$, $\boldsymbol{\Omega}_{\mathbf{2}}=\left[\Omega_{1,1}, \Omega_{1,2}, \Omega_{2,1}, \Omega_{2,2}\right]=[-6,-3,0,3] \mathrm{dB}$, and iv) $K_{R}^{(i, l)}=$ $K_{R}=0 \mathrm{~dB}$ for $i=1,2, \ldots, N_{t}$ and $l=1,2, \ldots, N_{r}$.

similar to the comment in ii). iv) Finally, by studying the performance with respect to the number of transmit- and receive-antennas we observe that the ABEP degrades for increasing values of $N_{t}$, while it gets better for increasing values of $N_{r}$. The trend obtained with respect to $N_{t}$ is similar to the performance degradation observed in digital modulation schemes with increasing values of the modulation order: This agrees with (21). Furthermore, numerical results confirm that the diversity order increases with $N_{r}$, as shown in Section III-C.

c) Performance of SSK Modulation over Rician Fading - Power Imbalance: In Figs. 5, 6, we study the performance of SSK modulation by considering a wireless scenario with non-identically distributed fading (i.e., with power imbalance among the wireless links). Besides the good agreement between Monte Carlo simulations and the analytical framework in Section III, we notice that the performance of SSK modulation changes remarkably for various fading scenarios. In particular, in Fig. 5 we observe two scenarios with a high (i.e, $\Omega_{2,1}-\Omega_{1,1}=10 \mathrm{~dB}$ ) and a low (i.e, $\Omega_{2,1}-\Omega_{1,1}=3 \mathrm{~dB}$ ) power imbalance between the wireless links. We notice that when the fading fluctuations are not very pronounced (i.e., $K_{R}=5 \mathrm{~dB}$ ), the ABEP improves with channel correlation, and the improvement increases with the average power gap $\Omega_{2,1}-\Omega_{1,1}$. This trend follows from (12) and (21): Although the channel fading might introduce fluctuations that could offset the power imbalance of the channel gains, if the wireless links are correlated these fluctuations are likely to be almost the same over all wireless links, thus unlikely offsetting the average power gap. On the contrary, if the wireless links are identically distributed (as shown in Figs. 1-4) channel fading can only be beneficial if it makes the wireless links more distinguishable from each other, and so, in this case, channel correlation can only be detrimental. These conclusions agree with the results already obtained in [9], [10] for Nakagami$m$ fading, thus showing that this property is inherent in SSK modulation and is not specific of the channel fading model. In Fig. 6, we show a setup similar to Fig. 5, but in this case the channels are subject to more pronounced fading fluctuations (i.e., $K_{R}=0 \mathrm{~dB}$ instead of $K_{R}=5 \mathrm{~dB}$ as in Fig. 5). We notice that in Fig. 6 the ABEP gets worse for increasing values of the channel correlation even though there is a nonnegligible power imbalance among the wireless links. The reason is that the deep fluctuations introduced by Rayleigh fading $\left(K_{R}=0 \mathrm{~dB}\right)$ offset the average power gap in this case. However, with respect to Figs. 1-4, we observe that power imbalance reduces, in any case, the effect of fading fluctuations and, so, the ABEP improves. These results clearly show that the performance of SSK modulation over generalized fading channels is very unpredictable, and the development of general and sound frameworks, as those derived in this paper, are very important for the optimization of this novel transmission technique.

\section{CONCLUSIONS}

In this paper, we have studied the performance of SSK modulation over correlated Rician fading channels. An accurate analytical framework has been developed, and numerical results have clearly indicated that the performance of SSK modulation is very unpredictable and strongly depends on the actual channel conditions. Furthermore, we have shown that conventional SSK modulation schemes are unable to offer transmit-diversity gains, which might limit their usefulness for downlink settings. Ongoing research is concerned with the development of improved SSK modulation schemes offering transmit-diversity gains.

\section{ACKNOWLEDGMENT}

We gratefully acknowledge support from the EPSRC (EP/G011788/1) for this work. Harald Haas acknowledges the 
Scottish Funding Council support of his position within the Edinburgh Research Partnership in Engineering and Mathematics between the University of Edinburgh and Heriot Watt University.

\section{REFERENCES}

[1] Y. Chau and S.-H. Yu, "Space modulation on wireless fading channels", IEEE Veh. Technol. Conf., vol. 3, pp. 1668-1671, Oct. 2001.

[2] H. Haas, E. Costa, and E. Schultz, "Increasing spectral efficiency by data multiplexing using antennas arrays", IEEE Int. Symp. Personal, Indoor, Mobile Radio Commun., vol. 2, pp. 610-613, Sept. 2002.

[3] R. Y. Mesleh, H. Haas, S. Sinanovic, C. W. Ahn, and S. Yun, "Spatial modulation", IEEE Trans. Veh. Technol., vol. 57, no. 4, pp. 2228-2241, July 2008.

[4] J. Jeganathan, A. Ghrayeb, and L. Szczecinski, "Spatial modulation: Optimal detection and performance analysis", IEEE Commun. Lett., vol. 12, no. 8, pp. 545-547, Aug. 2008.

[5] J. Jeganathan, A. Ghrayeb, L. Szczecinski, and A. Ceron, "Space shift keying modulation for MIMO channels", IEEE Trans. Wireless Commun., vol. 8, no. 7, pp. 3692-3703, July 2009.

[6] L. Xiao, L. Greenstein, N. Mandayam, and W. Trappe, "Using the physical layer for wireless authentication in time-variant channels", IEEE Trans. Wireless Commun., vol. 7, no. 7, pp. 2571-2579, July 2008.

[7] A. Alshamali and B. Quza, "Performance of spatial modulation in correlated and uncorrelated Nakagami fading channel", J. Commun., vol. 4, no. 3, pp. 170-174, Apr. 2009.

[8] J. Jeganathan, A. Ghrayeb, and L. Szczecinski, "Generalized space shift keying modulation for MIMO channels", IEEE Int. Symp. Personal, Indoor, Mobile Radio Commun., pp. 1-5, Sept. 2008.

[9] M. Di Renzo and H. Haas, "A general framework for performance analysis of space shift keying (SSK) modulation for MISO systems over correlated Nakagami- $m$ fading channels", IEEE Trans. Commun., Jan. 2010 (accepted pending minor revision).

[10] M. Di Renzo and H. Haas, "Space shift keying (SSK) modulation with partial channel state information at the receiver: Optimal detector and performance analysis over correlated fading channels", IEEE Trans. Commun., Jan. 2010 (accepted pending minor revision).
[11] P. G. Moschopoulos, "The distribution of the sum of independent gamma random variables", Ann. Inst. Statist. Math. (Part A), vol. 37, pp. 541544,1985

[12] M. Abramowitz and I. A. Stegun, Handbook of Mathematical Functions with Formulas, Graphs, and Mathematical Tables, New York, Dover, 9th ed., 1972.

[13] A. P. Prudnikov, Y. A. Brychkov, and O. I. Marichev, Integrals and Series. Vol. 3: More Special Functions, 2003.

[14] M. K. Simon and M.-S. Alouini, Digital Communication over Fading Channels: A Unified Approach to Performance Analysis, John Wiley \& Sons, Inc., 1st ed., 2000.

[15] H. L. Van Trees, Detection, Estimation, and Modulation Theory, Part I: Detection, Estimation, and Linear Modulation Theory, John Wiley \& Sons, Inc. 2001, ISBNs: 0-471-09517-6.

[16] D. Tse and P. Viswanath, Fundamentals of Wireless Communication, Cambridge University Press, 2005.

[17] J. Cheng and T. Berger, "Capacity of a class of fading channels with channel state information (CSI) feedback", Allerton Conference on Communication, Control, and Computing, pp. 1152-1160, Oct. 2001.

[18] G. Golub and J. Welsch, "Calculation of gauss quadrature rules", Math. Comput., vol. 23, pp. 221-230, Apr. 1969.

[19] M.-S. Alouini, A. Abdi, and M. Kaveh, "Sum of gamma variates and performance of wireless communication systems over Nakagami- $m$ fading channels", IEEE Trans. Veh. Technol., vol. 50, no. 6, pp. 14711480, Nov. 2001

[20] Z. Wang and G. B. Giannakis, "A simple and general parameterization quantifying performance in fading channels", IEEE Trans. Commun., vol. 51, no. 8, pp. 1389-1398, Aug. 2003.

[21] J. P. Kermoal, L. Schumacher, K. I. Pedersen, P. E. Mogensen, and F. Frederiksen, "A stochastic MIMO radio channel model with experimental validation", IEEE J. Sel. Areas Commun., vol. 20, no. 6, pp. 1211-1226, Aug. 2002.

[22] W. Weichselberger, M. Herdin, H. Özcelik, E. Bonek, "A stochastic MIMO channel model with joint correlation of both link ends", IEEE Trans. Wireless Commun., vol. 5, no. 1, pp. 90-100, Jan. 2006.

[23] M. Chiani, M. Z. Win, and A. Zanella, "On the capacity of spatially correlated MIMO Rayleigh-fading channels", IEEE Trans. Inform. Theory, vol. 49, no. 10, pp. 2363-2371, Oct. 2003 